\title{
Editorial:
}

\section{Jahre Schweizerische Gesellschaft für Geschichte der Medizin und der Naturwissenschaften}

Marcel H. Bickel

Hans Fischer gedachte in Gesnerus 1971 in informativer Weise des fünfzigjährigen Bestehens der Schweizerischen Gesellschaft für Geschichte der Medizin und der Naturwissenschaften (SGGMN) ${ }^{1}$, der Herausgeberin dieser Zeitschrift. Wenn eine weitere Erinnerung schon im wenig «runden» 75. Jahr folgt, so vor allem wegen der Anwesenheit einer neuen Generation von Mitgliedern und am Fach Interessierten. Ausserdem weiss jeder Historiker, dass die Sicht der Dinge sich in 25 Jahren ändert, Geschichte also nicht nur nachgeführt, sondern auch nach-gedacht werden muss.

\section{Gründung}

Die 1921 erfolgte Gründung der SGGMN ist nach den Worten von H. Fischer «mehr oder weniger in Dunkel gehüllt $»^{2}$. H. E. Sigerist nennt Arnold C. Klebs als Gründer der Gesellschaft ${ }^{3}$; korrekter ist wohl, von einem Kollektiv von Gründern zu sprechen, zu welchem auch E. Olivier, G. Senn, H. E. Sigerist, J. Strohl, R. von Fellenberg und W. E. von Rodt gehörten ${ }^{4}$. Wenn sich unter ihnen kein Professor der Medizin- oder Wissenschaftsgeschichte befand, so deshalb, weil dieses Fach in der Schweiz noch kaum anerkannt, geschweige denn institutionalisiert war. Daher auch die zeitliche Verzögerung gegenüber der 20 Jahre früher erfolgten Gründung der Deutschen Gesellschaft für

1 Hans Fischer: 50 Jahre Schweizerische Gesellschaft für Geschichte der Medizin und der Naturwissenschaften, Gesnerus 28: 72-78, 1971.

2 (n. 1), p. 72.

3 H. E. Sigerist: Gesnerus [Anzeige der Gründung], Bulletin of the History of Medicine 17: 209-210, 1945 .

4 (n. 1) p. 72.

Prof. Dr. M. H. Bickel, Medizinhistorisches Institut, Postfach, CH-3000 Bern 9 
Geschichte der Medizin und der Naturwissenschaften. ${ }^{5}$ Die Gründer der schweizerischen Gesellschaft waren Privatdozenten der Medizingeschichte, historisch interessierte Naturwissenschaftler, Autodidakten.

An Stelle eines Tätigkeitsberichts für das Gründungsjahr 1921 lesen wir folgende Absichtserklärung:

«Im Wunsche, die historisch interessierten Aerzte und Naturforscher der Schweiz zu vereinigen, wurde am 4. Dezember 1921 in Bern die Schweizerische Gesellschaft für Geschichte der Medizin und der Naturwissenschaften gegründet. Auf die erste Einladung hin traten ihr 120 Mitglieder bei, ein Zeichen, dass die Gesellschaft einem Bedürfnis entgegenkommt. Ausser der Jahresversammlung sieht die Gesellschaft auch lokale Versammlungen vor und wird jährlich eine Arbeit aus dem Gebiet der Geschichte der Medizin oder der Geschichte der Naturwissenschaften veröffentlichen. Der Jahresbeitrag wurde auf Fr. 10 festgesetzt».

Der Sekretär: Dr. Henry E. Sigerist» ${ }^{6}$.

Neben ihrer Funktion als Interessengemeinschaft waren also die wichtigsten Anliegen der SGGMN von Anfang an die Abhaltung von Tagungen zum wissenschaftlichen Informationsaustausch sowie die Herausgabe von $\mathrm{Pu}-$ blikationen zum Fachgebiet. Erst später kam dazu die Herausgabe einer eigenen Fachzeitschrift.

\section{Fachtagungen und Organisation der SGGMN}

Die SGGMN wurde gleich nach ihrer Gründung als zwölfte Fachgesellschaft in die Schweizerische Naturforschende Gesellschaft (SNG) ${ }^{7}$ als ihre Dachorganisation aufgenommen und beteiligte sich fortan an deren Jahrestagungen. Die SGGMN tagte erstmals 1922 in Bern, dann jährlich mindestens einmal an zahlreichen Orten der Schweiz. Einzig im Herbst 1939 entfiel die Tagung infolge der Wirren nach Ausbruch des Zweiten Weltkriegs und der Mobilmachung der schweizerischen Armee. An den ersten Tagungen konnte es vorkommen, dass nur drei Vortragende vor 15 Zuhörern sprachen. Später wurden oft 20 und mehr Vorträge und 50-100 Teilnehmer verzeichnet. Schon früh gab es neben freien Vorträgen auch thematische Symposien, Gemeinschaftstagungen mit anderen wissenschaftlichen Gesellschaften, ausserplanmässige Tagungen, auch Gedenkfeiern, Besichtigungen, Ausstellungen ${ }^{8}$.

5 H. H. Eulner: Die Entwicklung der medizinischen Spezialfächer an den Universitäten des deutschen Sprachgebiets.

Ferdinand Enke, Stuttgart, 1970. p. 438.

6 Verhandlungen der Schweizerischen Naturforschenden Gesellschaft. I.Teil, p. 104, 1922.

7 Seit 1988 Schweizerische Akademie der Naturwissenschaften (SANW).

8 Die Akten der SGGMN befinden sich im Medizinhistorischen Institut der Universität Zürich. Knappe Jahresberichte und Zusammenfassungen der Vorträge in den Verhandlungen der SNG, ab 1978 Jahrbücher der SNG, ab1988 Jahrbücher der SANW (siehe n. 7). 
Zur Aufrechterhaltung solcher Aktivitäten bedarf eine wissenschaftliche Gesellschaft der organisatorischen Tätigkeit durch den von ihr gewählten Vorstand. Anhang I führt die Vorstandsmitglieder der ersten 75 Jahre auf, in der Meinung, dass diese in der Regel besonders motivierte Mitglieder der Gesellschaft sind oder aber infolge ihrer wissenschaftlichen Leistungen gewählt werden. Neben den oben genannten Gründungsmitgliedern erwähnt H. Fischer für die Frühzeit der SGGMN noch besonders G. A. Wehrli, W. H. Schopfer und B. Milt ${ }^{9}$. Ganz besondere Erwähnung verdient jedoch der Chronist der ersten 50 Jahre selbst, Hans Fischer, der neben seiner Stellung als Ordinarius der Pharmakologie während über dreissig Jahren zusammen mit fünf Präsidenten als Sekretär und Quästor die Geschicke der SGGMN leitete. Die auf den Zweiten Weltkrieg folgenden Jahrzehnte waren dann geprägt durch Medizin- und Wissenschaftshistoriker/innen wie E. H. Ackerknecht, H. Buess, H. M. Koelbing, J. Starobinski, E. Fischer-Homberger und andere. Eine Würdigung der jetzigen Generation der Hauptvertreter wird Sache späterer Chronisten sein.

Die Mitglieder der SGGMN repräsentierten stets ein weites berufliches Spektrum von Ärzten und Wissenschaftlern, die sich für die Geschichte ihres Fachs interessieren. Die Zahl von 120 Mitgliedern, die der SGGMN schon bei der Gründung beigetreten sind, ist erstaunlich hoch für eine Zeit ohne institutionalisierte Medizin- und Wissenschaftsgeschichte. Die Mitgliederzahl erhöhte sich 1956 auf 200,1982 auf 300 und 1996 auf 350.

\section{Publikationen. Zusätzliche Aktivitäten}

Wie schon erwähnt, war von Anfang an auch eine publizistische Tätigkeit der SGGMN vorgesehen, und zwar in Form von Monographien, welche im Abstand von ungefähr einem Jahr erscheinen sollten. Bis heute sind 44 dieser Veröffentlichungen der SGGMN erschienen (Anhang II a) ${ }^{10}$. Der Autor der ersten dieser Monographien, Conrad Brunner, stellte seinem Buch über mittelalterliche Medizin in der Schweiz folgende Widmung voran:

9 (n. 1), p. 73-76.

10 Veröffentlichungen der Schweizerischen Gesellschaft für Geschichte der Medizin und der $\mathrm{Na-}$ turwissenschaften Nr. 1 (1922) bis Nr. 40 (1987). Fortgesetzt als Gesnerus Supplementa Nr. 41 (1990) bis Nr. 44 (1995). Titelverzeichnis im Gesamtverzeichnis Bücher und Zeitschriften von Schwabe \& Co. AG, Basel, ab 1995/96; Titel vor 1946 in H. Buess: Die historischen Grundlagen der intravenösen Injektion. Veröffentlichungen der SGGMN Nr. 15 (1946). 
«Der neugegründeten Schweizerischen Gesellschaft für Geschichte der Medizin und Naturwissenschaften, die sich die Erforschung der schweizerisch-medizinischen Vergangenheit mit zur Aufgabe macht, sei diese Studie mit den besten Wünschen für die Zukunft als ein Zeichen der Sympathie gewidmet vom Verfasser» ${ }^{11}$

1943 wurde unter der Bezeichnung Gesnerus die Zeitschrift der SGGMN gegründet. Es war ein Zeichen dafür, dass in der Schweiz eine hinreichende Zahl von Autoren medizin- und wissenschaftshistorischer Studien existierte. Gründer dieser Zeitschrift waren A. C. Klebs, J. Strohl und H. Fischer ${ }^{12}$. Während die beiden ersten das Erscheinen der Zeitschrift nicht mehr erlebten, war es Fischer, der sie als Redaktor während nicht weniger als 30 Jahren gestaltete. Damit ist evident, dass keine Einzelperson für die SGGMN so viel geleistet hat als ihr langjähriger Sekretär, Quästor und Redaktor Hans Fischer. Seine umsichtige Leitung der neuen Zeitschrift war gerade aus finanziellen Gründen keine leichte Aufgabe. Die Erhöhung des Mitgliederbeitrags von Fr. 10.- auf Fr. 12.- (heute Fr. 65.-) konnte die Druckkosten bei weitem nicht decken. Ermöglichten anfänglich private und andere $\mathrm{Zu}$ wendungen ein Überleben ${ }^{13}$, so ist es seit den 60er Jahren die Schweizerische Akademie der Naturwissenschaften (damals SNG), welche das Publikationsorgan ihrer Tochtergesellschaft mit substantiellen Zuwendungen unterstützt. Der gelehrte Zürcher Arzt und Naturwissenschaftler Conradus Gesnerus (1516-1565) als Vorbild für die Gründer dieser Zeitschrift hatte zu deren Namen geführt. Im Zuge ihrer zunehmenden Bekanntheit auf der internationalen Bühne erhielt sie 1988 die Zusatzbezeichnung Swiss Journal of the History of Medicine and Sciences. Im folgenden Jahr wurde ihre Leitung zu einem Redaktionskollegium mit einem Chefredaktor erweitert, und 1993 konnte der 50. Jahrgang erscheinen ${ }^{14}$, gefolgt von einem Index, welcher diese ersten 50 Bände erschliesst ${ }^{15}$.

Eine wesentliche Bereicherung erfuhr die SGGMN durch die Errichtung einer Dr-Markus-Guggenheim-Schnurr-Stiftung durch den Basler Biochemiker im Jahr 1962. Sie «dient der Förderung und Verbreitung wissenschaftlicher Arbeiten auf den Gebieten der Geschichte der Medizin und der Naturwissenschaften ${ }^{16}$ durch Ausrichtung von Druckkostenbeihilfen und der Unterstützung von Gesnerus. Dank ihrer Mittel findet anlässlich der SGGMN-

11 Conrad Brunner: Ueber Medizin und Krankenpflege im Mittelalter in Schweizerischen Landen. Seldwyla, Zürich 1922.

12 J. Strohl, H. Fischer: Zur Einführung. Gesnerus 1: 1-4, 1943.

13 Schweizerische Akademie der medizinischen Wissenschaften, Ciba AG, Stiftung Pro Helvetia, Schweizerischer Nationalfonds zur Förderung der wissenschaftlichen Forschung.

14 M. H. Bickel: Editorial. Gesnerus (Swiss J. Hist. Med. Sci.) 50: 6-10,1993.

15 Gesnerus Index Vol. 1-50 1943/44-1993. Supplementum Gesnerus 44 (1995), Schwabe \& Co. AG, Verlag, Basel.

16 Stiftungsurkunde, $\S 3$. 
Jahrestagungen der Guggenheim-Vortrag statt. Wie aus Anhang IIb hervorgeht, werden dafür prominente schweizerische und internationale Medizinund Wissenschaftshistoriker als Referenten eingeladen. Viele unter ihnen sind auch Ehrenmitglieder der SGGMN. Seit 1968 wird aus Mitteln der Guggenheim-Stiftung der Henry E. Sigerist-Preis für herausragende Dissertationen auf dem Gebiet der Medizingeschichte ausgerichtet. Die 33 bisherigen Preisträgerinnen und Preisträger (Anhang IIc) zeugen von dieser Form der Nachwuchsförderung durch die SGGMN.

Dass eine kleine Gesellschaft für ein kleines Fachgebiet internationaler Ausrichtung bedarf, scheint von Anbeginn klar gewesen zu sein. Wichtige Vertreter der Medizin- und Wissenschaftsgeschichte wurden schon bald nach der Gründung der SGGMN zu Ehrenmitgliedern ernannt: Sudhoff in Deutschland, Neuburger in Österreich, Garrison in den USA, Singer in England, und natürlich auch das in Leipzig und Baltimore zu internationaler Bedeutung gelangte Gründungsmitglied H. E. Sigerist. Bereits 1928 trat die SGGMN der Internationalen Gesellschaft für Geschichte der Medizin bei, 1948 der Union internationale d'histoire des sciences. Lange vor der Errichtung der Guggenheim-Vorträge wurden schon ausländische Referenten eingeladen und Symposien mit internationaler Beteiligung durchgeführt.

Natürlich gab es in der Schweiz auch medizinhistorische Aktivitäten ausserhalb der SGGMN. Dazu gehörten zeitweilig die regelmässig im Herbst in Aarau stattfindenden Diskussionen der schweizerischen Dozenten der Medizingeschichte ${ }^{17}$. Ein an der Jahrestagung 1949 der SGGMN ohne Diskussionen durchgepeitschtes Vortragsprogramm dürfte H. E. Sigerist veranlasst haben, ab 1950 die jährlichen Pura-Konferenzen zu organisieren ${ }^{18}$. Sie wurden nach seinem Tod als $H$. E. Sigerist-Tagungen einige Jahre fortgeführt. Ausserhalb der Tätigkeiten der SGGMN lag auch die Herausgabe der Basler, Berner und Zürcher Reihen medizinhistorischer Veröffentlichungen.

\section{Institutionalisierung der Medizingeschichte}

Weitgehend ausserhalb der SGGMN, doch von ihr beeinfluss $\mathrm{t}^{19}$, vollzog sich schliesslich die Etablierung der Medizingeschichte an den schweizerischen Universitäten. Das Interesse an der Geschichte der Medizin und der Natur-

17 H. E. Sigerist: The History of Medicine in Switzerland. Bull. Hist. Med. 26: 187-190, 1952.

18 H. E. Sigerist: Letter from Pura. Bull. Hist. Med. 24: 77-83, 1950.

19 Die Statuten der SGGMN von 1971 enthalten neu den Satz «Sie [die SGGMN] sucht den Unterricht und die Forschung in der Geschichte dieser Fächer [Medizin und Naturwissenschaften] an den schweizerischen Universitäten zu verankern». (\$2) 
wissenschaften ist nach einer Phase der Verdrängung durch wissenschaftliche Fortschrittseuphorie erst im 20. Jahrhundert wieder erwacht. In den ersten Jahrzehnten entstanden die ersten Professuren und Institute im deutschen Sprachraum - zuerst in Leipzig und Wien - ferner auch die ersten Fachzeitschriften. Mit diesen Elementen war die eigentliche akademische Institutionalisierung eingeleitet. Weitere Institute folgten im Verlauf der ersten Hälfte des Jahrhunderts. Die Schweiz hat diese Entwicklung nachvollzogen, wenn auch mit der üblichen Verspätung. Zürich schritt 1951 voran mit der Errichtung einer Professur und der Umwandlung der medizinhistorischen Sammlung von G. A. Wehrli zum Institut. Dieser Durchbruch ist wiederum in hohem Masse das Verdienst von Hans Fischer, der - nominell nicht als Vertreter der SGGMN, sondern als Dekan der Medizinischen Fakultät - in seinem Gutachten 1949 unter anderem zu bedenken gab, es solle

\begin{abstract}
«der angehende Arzt Gelegenheit erhalten, in die Geschichte seines Faches eingeführt und über Lehre und Irrlehre medizinischer Vergangenheit und Gegenwart orientiert zu werden. Dabei handelt es sich nicht in erster Linie um die Uebermittlung interessanter medizin- und kulturhistorischer Facta - so reich, ja überreich die Medizingeschichte an solchen ist - sondern um die Vermittlung des ideellen Werdeganges und die besonderen geistigen Grundlagen der wissenschaftlichen Medizin, um Fragen der Grundstruktur des ärztlichen Denkens, um die Geschichte der Stellung des Arztes im sozialen Aufbau der Gesellschaft bis zur Gegenwart. So aufgefasst und vertreten, liefert die Medizingeschichte einen wertvollen Beitrag zur Ideen- und Kulturgeschichte überhaupt und bildet ein wertvolles Bindeglied zwischen den Geisteswissenschaften und einer vielleicht allzu materialistisch gewordenen Medizin. Die medizinische Fakultät verspricht sich vom medizingeschichtlichen Unterricht eine sehr wünschbare Horizonterweiterung des angehenden Arztes, welche auf die Entwicklung seiner Persönlichkeit von wohltätigstem Einfluss sein kann» ${ }^{20}$.
\end{abstract}

Die erste Professur in Zürich und damit in der Schweiz bekleidete B. Milt. Ihm folgten E. H. Ackerknecht, H. M. Koelbing und B. Rüttimann. Aus der Wehrlischen Sammlung wurde um 1990 ein Medizinhistorisches Museum von internationaler Bedeutung. Die nächste Errichtung einer medizinhistorischen Professur erfolgte über ein Vierteljahrhundert nach der ersten in Bern (1978 E. Fischer-Homberger, 1985 U. Boschung). Die dort von E. Hintzsche aufgebaute Bibliothek diente als Nukleus für das 1979 gegründete Institut. Unüblicherweise war die Westschweiz auf diesem Gebiet noch langsamer, indem Professuren und Institute erst um 1990 gegründet wurden: In Genf das Institut Louis Jeantet d'histoire de la médecine (B. Fantini), in Lausanne das Institut d'histoire de la médecine et de la santé publique (G. Saudan, gefolgt von V. Barras) ${ }^{21}$. Die beiden Institute wurden 1995 zum Institut romand d'histoire de la médecine et de la santé vereinigt.

20 Zitiert nach H. M. Koelbing und U. Boschung: Das Medizinhistorische Institut der Universität Zürich. Zürcher Taschenbuch 1976. Buchdruckerei a/d Sihl, Zürich, 1975, p. 40-58.

21 Université de Genève, Faculté de Médecine: Institut Louis Jeantet d'histoire de la médecine. Rapport d'activité (1990-1994). Genève 1995. 
Die SGGMN ist eine kleine Gesellschaft für ein kleines wissenschaftliches Fach in einem kleinen Land. Kleinheit vereinfacht manches, verpflichtet aber auch, wenn das qualitativ Wesentliche nicht auch klein bleiben soll.Ein Rückblick, wie der hier versuchte, mag eine Antwort auf die Frage erlauben, ob denn die SGGMN ihre Zielsetzungen von 1921 erfüllt habe. Selbst bei nüchterner Betrachtung scheint hier eine überwiegend positive Antwort angezeigt. Die SGGMN hat zu allen Zeiten zahlreiche Aktivitäten stimuliert, kanalisiert und koordiniert, sie hat sich Sympathie und Unterstützung erworben, sie hat vor allem die Qualität ihrer wissenschaftlichen Aktivitäten und ihrer literarischen Produkte hochzuhalten verstanden, was zu Ausstrahlung und internationaler Anerkennung geführt hat. Dies ist nicht das Verdienst einer anonymen Institution, sondern fast ausschliesslich der sie führenden und stützenden Persönlichkeiten, unter welchen einige internationale Spitzenvertreter des Fachs zu finden sind. Jede Organisation kann nur so gut sein wie die sie vertretenden Mitglieder. Es ist zu hoffen, dass auch das nächste Vierteljahrhundert trotz beengender wirtschaftlicher Verhältnisse eine gedeihliche Entwicklung zulassen wird. 


\section{Anhang I \\ Mitglieder des Vorstands der SGGMN}

Präsidenten

1921-35 G. Senn

1935-42 J. Strohl

1942-47 R. von Fellenberg

1948-61 W. H. Schopfer

1961-71 E. H. Ackerknecht

1972-77 H. Buess

1978-82 H. M. Koelbing

1983-84 E. Fischer-Homberger

1985-86 R. Joris

1987-89 M. H. Bickel

1990-92 C. Müller

1993-95 J. J. Dreifuss

1996- U. Boschung

Sekretäre/Quästoren

1921-25 H. E. Sigerist

1925-28 G. A. Wehrli

1928-35 A. Voirol

1935-69 H. Fischer

1969-77 H. M. Koelbing

1977-85 A. Gubser

1986-95 U. Boschung

1996- S.: C. Mörgeli, Q.: I. Ritzmann

Beisitzer

F. Sarasin, 1921-25

A. C. Klebs, 1921-35

W. E. von Rodt, 1921-41

C. C. Cumston, 1921-28

J. Strohl, 1924-27

O. Bernhard, 1925-39

G. A. Wehrli, 1942-49

R. von Fellenberg, 1942, 48-59

W. H. Schopfer, 1943-45

F. Nager, 1943-59

C. Baehny, 1946-47

B. Milt, 1949-56

H. Buess, 1955-66

E. H. Ackerknecht, 1957-61

C. Lichtenthaeler, 1962-66

E. Hintzsche, 1964-68

H. M. Koelbing, 1966-69

H. Balmer, 1969-80
Vizepräsidenten

1921-45 A. Guisan

1945-47 W. H. Schopfer

1947-64 C. Baehny

1966-71 H. Buess

1972-77 E. H. Ackerknecht

1978-80 J. Starobinski

1981-82 E. Fischer-Homberger

1982-83 R. Joris

1985-89 J. Starobinski

1990-92 J. J. Dreifuss

1993-95 B. Rüttimann

1996- P. Mudry

Redaktoren/Chefredaktoren*

1928-41 G. A. Wehrli

1943-73 H. Fischer

1974-80 H. H. Walser

1976-82 H. Balmer

1982-88 C. Haffter

1989-92 H. M. Koelbing*

1993- M. H. Bickel**

P. Eichenberger, 1972-77

P. Joris, 1975-81, 1987

J. Starobinski, 1976-77, 82-84, 90-92

E. Fischer-Homberger, 1978-80

H. H. Walser, 1982-84

U. Tröhler, 1982-95

M. Archinard, 1984-88

U. Boschung, 1985

M. H. Bickel, 1986, 90-92

B. Rüttimann, 1987-92, 96-

J. J. Dreifuss, 1989, 96-

H. K. Schmutz, 1989-

C. Müller, 1989, 93

P. Mudry, 1993-95

B. Fantini, 1993-

C. Mörgeli, 1995

M. Buscaglia, 1996- 


\section{Anhang II}

a) Autoren der Supplementa Gesnerus (vormals Veröffentlichungen der SGGMN)

\begin{tabular}{|c|c|c|c|c|}
\hline 11922 & C. Brunner & 161947 & H. Nigst & 311978 R. Schmid \\
\hline 21923 & G. A. Wehrli & 171947 & H. Buscher & 321979 S. Richter \\
\hline 31924 & O. Bernhard & 181949 & G. Reichen & 331979 H. M. Koelbing \\
\hline 41925 & A. Troendle & 191951 & S. Bornhauser & 341980 P. Кöpp \\
\hline 51926 & O. Bernhard & 201956 & H. Balmer & 351981 A. Faller \\
\hline 61928 & Peyer/Remund & 211957 & H. H. Walser & 361981 C. Prestele \\
\hline 71930 & A. Guisan & 221958 & E. Frenk & 371981 C. Picco \\
\hline 81933 & G. Senn & 231962 & A. Boner & 381982 H. K. Iselin \\
\hline 91934 & Bloch/Lang & 241970 & H. H. Walser & 391985 R. Neiger \\
\hline 1936 & de Quervain/Blösch & 251971 & H. Reverdin & 401987 A. H. Maehle \\
\hline 1938 & Aebischer/Olivier & 261973 & U. Tröhler & 411990 A. Schett \\
\hline 1941 & E. Fueter & 271974 & G. Schüler & 421990 H. W. Schefer \\
\hline 1943 & H. Fischer & 281974 & A. Kleiner & 431993 Kupferschmid \\
\hline 1946 & P. Niggli & 291976 & B. C. Hansch-Mock & 441995 Bickel/Seger/ \\
\hline 1946 & H. Buess & 301976 & K. Wäckerlin & Boschung/Wilhelm \\
\hline
\end{tabular}

b) Dr-Markus-Guggenheim-Schnurr-Gedenkvorträge

1963 J. Starobinski, Genève

1964 R. Hoykaas, Zeist, NL

1965 G. Petit, Paris

1966 E. Lesky, Wien

1967 G. Rudolph, Kiel

1968 W. Löffler, Zürich

1969 K. E. Rothschuh, Münster

1970 H. Beck, Bonn

1971 E. H. Ackerknecht, Zürich

1972 G. Majno, Genève

1973 H. Balmer, Bern

1974 N. Mani, Bonn

1975 H. Zoller, Basel

1976 J. Starobinski, Genève

1977 M. Schramm, Tübingen

1978 H. Schadewaldt, Düsseldorf

1979 M. Mattmüller, Basel
1980 A. H. Murken, Aachen

1981 L. Belloni, Milano

1982 R. Joly, Bruxelles

1983 M. J. Imbault-Huard, Paris

1984 H. M. Koelbing, Zürich

1985 H. H. Walser, Zürich

1986 L. Premuda, Padova

1987 W. F. Bynum, London

1988 M. E. Müller, Bern

1989 P. Mudry, Lausanne

1990 M. D. Grmek, Paris

1991 D. Kuhn, Marbach

1992 H. Mollaret, Paris

1993 H. Schipperges, Heidelberg

1994 D. B. Weiner, Los Angeles

1995 G. Charpak, Paris 
c) Henry E. Sigerist-Preisträger

1968 P. Wobmann

1981 R. Mazzolini

1969 H. P. Schönwetter

1982 F. Hugli

1970 H. Haefliger

1983 M. Nicola

1971 U. Bueck-Rich, F. Kubli

1984 C. Ducommun

1972 W. F. Steinebrunner

1985 P. Müller

1973 C. Montandon

1986 C. Bachmann

1974 E. Neuenschwander, U. Tröhler

1987 D. Heinrich

1975 A. Conti, C. Geduldig

1988 L. Welker

1976 A. Pfarrwaller

1989 R. Neff, B. Y. Bohner

1977 M. Hämmerle

1990 S. Brändli

1978 R. Schmid

1991 J. Willi

1979 F. Ledermann

1992 J. Gasser

1980 G. Heller, P. Meerwein

1993 H. Kupferschmidt

1994 M.-F. Vouilloz Burnier 